\title{
La economía popular y solidaria. Una alternativa de desarrollo económico local en el caso del cantón cuenca
}

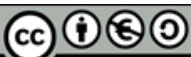

\author{
The popular and solidarity economy. An alternative for local economic \\ development in the case of the cuenca canton
}

\author{
Carabajo Alvear Román Florencio. ${ }^{1}$ \& Rosales Namicela Mónica Briggith. ${ }^{2}$
}

Recibido: 08-01-2021 / Revisado: 13-01-2021 /Aceptado: 07-02-2021/ Publicado: 05-03-2021

\begin{abstract}
DOI: https://doi.org/10.33262/concienciadigital.v4i1.2.1581

Introduction. The popular and solidarity economy in Ecuador was recognized in the 2008 Constitution, giving way to the approval of the Organic Law of the Popular and Solidarity Economy in 2011. The common needs of people and the demand for products, goods and services from The State generated the creation of new organizations that are part of the social fabric and productive sector, hence the need to identify them and know their characteristics, as well as their importance within the local development of a territory. Objective. Determine the characteristics of the non-financial sector of the popular and solidarity economy, in the case of the Cuenca canton. Methodology. The research is descriptive, cross-sectional and correlational in nature. To characterize the organizations of the popular and solidarity economy -OEPS, a questionnaire from the Guide for mapping and surveying the Popular and Solidarity Economy in Latin America and the Caribbean was applied to obtain and analyze information on three thematic axes: articulation of the actors of the popular solidarity economy and the social and solidarity economy, characteristics of the Popular Solidarity Economy organizations, and characteristics of the products, goods and services of the OEPS, finally to identify the relationship between the variables number of members and monthly income. Results. In the Cuenca canton there are 308 OEPS, $86 \%$ in active status: 151 are associations being $57 \%, 113$ are cooperatives being $42 \%$, and 2 unions being $1 \% .65 \%$ of the OEPS are urban and $35 \%$ rural. 18 classes of associations, 11 types of cooperatives and 1 class of

\footnotetext{
${ }^{1}$ Afiliación: Carabajo Alvear Román Florencio, Universidad Católica de Cuenca, Maestría en Desarrollo Local Mención en Ordenamiento Territorial, Azuay, rcalvear77@gmail.com https://orcid.org/0000-00019270-714X

2 Afiliación: Rosales Namicela Mónica Briggith, Universidad Católica de Cuenca, Carrera de Economía, Azuay, mrosales@ucacue.edu.ec https://orcid.org/0000-0002-3240-1146
} 
union are identified. Conclusion. The reliability analysis presented a Cronbach's alpha coefficient of 0.881 . The results of the analysis of the variables show that the economic income of the organizations is higher in the organizations that have a greater number of members.

Keywords: local development, popular and solidarity economy, social and solidarity economy

\section{Resumen.}

Introducción. La economía popular y solidaria en el Ecuador fue reconocida en la Constitución 2008, dando paso a la aprobación de la ley Orgánica de la Economía Popular y Solidaria en el 2011. Las necesidades comunes de las personas y la demanda de productos, bienes y servicios desde el Estado, generó que se constituyan nuevas organizaciones siendo parte del tejido social y sector productivo, por ello la necesidad de identificarlas y conocer sus características, así como su importancia dentro del desarrollo local de un territorio. Objetivo. Determinar las características del sector No financiero de la economía popular y solidaria, en el caso del cantón Cuenca. Metodología. La investigación es de naturaleza descriptiva, transversal y correlacional. Para caracterizar a las organizaciones de economía popular y solidario -OEPS se aplicó un cuestionario de la Guía para el mapeo y relevamiento de la Economía Popular y Solidaria en Latinoamérica y Caribe para obtener y analizar información de tres ejes temáticos: articulación de los actores de la economía popular solidaria y la economía social y solidaria, características de las organizaciones de Economía Popular Solidaria, y características de los productos, bienes y servicios de los OEPS, finalmente identificar la relación entre las variables número de socios/as y el ingreso mensual. Resultados. En el cantón Cuenca existen 308 OEPS, el 86\% en estado activo: 151 son asociaciones siendo el 57\%, 113 son cooperativas siendo el 42\%, y 2 uniones siendo 1\%. El 65\% de las OEPS son urbanas y $35 \%$ rurales. Se identifican 18 clases de asociaciones, 11 tipos de cooperativas y 1 clase de unión. Conclusión. El análisis de fiabilidad presentó un coeficiente Alpha de Cronbach 0,881. Los resultados de análisis de las variables evidencian que el ingreso económico de las organizaciones es mayor en las organizaciones que tienen mayor número de socios/as.

Palabras claves: desarrollo local, economía popular y solidaria, economía social y solidaria

\section{Introducción}

El desarrollo económico local incurre en el mejoramiento de la competitividad, aumenta el desarrollo sostenible y asegura la inclusividad del crecimiento. Si bien es cierto, por algún tiempo el tema de desarrollo económico local no se lo ha tomado en cuenta para desarrollar teorías, actualmente este contenido ha sido relevante para la elaboración de 
las mismas. El desarrollo económico local se puede definir como un proceso de crecimiento y cambio estructural que, mediante la utilización del potencial de desarrollo existente en el territorio, conduce a elevar el bienestar de la población de una localidad o una región. (CEPAL, 2009).

Alburquerque (2004) plantea que es importante también fomentar la creación de microempresas y PYMES, así como también la capacitación del capital humano. Las instituciones para el desarrollo económico local también son muy importantes, por ejemplo, las cooperativas, mutualistas, entre otras.

Como lo menciona la CEPAL (2009: 164) el desarrollo económico local exige una acción decidida de las instancias públicas territoriales, lo cual obliga a incorporar dicha dimensión en los actuales programas de fortalecimiento de los gobiernos locales. Para impulsar el desarrollo económico local no sólo es preciso utilizar mejor los recursos endógenos sino también aprovechar las oportunidades de dinamismo externo existentes. Otros puntos fundamentales que se deben dar para generar un desarrollo es el fomento de la asociatividad y la cooperación entre las microempresas y pequeñas empresas conformando empresas solidarias, es importante también el vincular las universidades regionales y los centros de investigación científica y tecnológica con los sistemas productivos locales.

\section{El papel de la asociatividad en el desarrollo local}

El modelo asociativo surge como uno de los mecanismos de cooperación, el cual persigue la creación de valor a través de la solución de problemas comunes originados fundamentalmente, por falta de escala. La asociatividad permite, a través de la implementación de estrategias colectivas y de carácter voluntario, alcanzar niveles de competitividad similares a los de empresas de mayor tamaño.

La economía popular y solidaria es una respuesta a las teorías y prácticas dominantes de la economía capitalista contemporánea. Ha sido denominada por Lazarini (2010), Razzeto (2007), Coraggio (2011), con distintos nombres como: "economía social y solidaria", "economía de la solidaridad”, "economía del trabajo", entre otros.

Según Singer (2000) su basamento central es la solidaridad como principio de orden ético y moral contrapuesto al individualismo posesivo y competitivo de la sociedad capitalista.

Laville (2009) dice que los núcleos organizativos de la economía solidaria es un universo heterogéneo de organizaciones sociales cuya articulación está basada en el trabajo equivalente y en los derechos igualitarios de sus miembros como las cooperativas, los clubes de ahorro e intercambio, las empresas bajo control obrero, los comedores populares y los nodos de trueque, entre muchas otras formas de asociación.

Lazarini (2010) plantea que la economía solidaria designa a su vez a un conjunto diverso de debates y producciones teóricas, críticas al modo de producción capitalista, que giran en torno a la visualización y puesta en valor de las experiencias de las organizaciones y 
redes que pertenecen a este espacio económico alternativo, a la vez que formulan análisis y propuestas programáticas que procuran sostener y profundizar tales experiencias.

Para algunos autores la economía solidaria representa el tercer sector de la economía en tanto que está conformada por organizaciones no-gubernamentales mantenidas por los sectores populares (Razeto, 1993) o es parte de un espacio heterogéneo vinculado al polo marginal de la economía (Quijano, 2014).

Para otros autores representa un modo de producción y distribución alternativo al capitalismo (Razeto, 2007). De igual forma, algunos partidarios de la economía solidaria la visualizan como un sistema económico cuyo fin último consiste en reemplazar al capitalismo (Coraggio J. L., 2007), mientras que otros la consideran meramente como una forma de economía mixta (Razeto, 1984).

Según (Quijano, 2014), a pesar de las importantes diferencias a lo interno de los debates sobre economía solidaria, es común el reconocimiento de las experiencias solidarias de producción, distribución e intercambio como prácticas sociales alternativas que han habitado históricamente en las fronteras de la hegemonía global del capital, relacionándose de manera desigual, contradictoria y discontinua con el capitalismo. La capacidad de perduración y de reproducción de las prácticas de economía solidaria, así como su expansión mundial y la magnitud de las poblaciones que en ella están implicadas, hacen considerar a estas experiencias como parte fundamental del movimiento actual de la sociedad global.

Dentro de esta coyuntura histórica se constituyen diversas organizaciones y experiencias de economía solidaria como modalidades de supervivencia, pero también como búsquedas de alternativas al capitalismo. A la par de este movimiento, reaparecen en la agenda de las ciencias sociales latinoamericanas los debates sobre las características y tendencias de los modos de supervivencia económica y de reproducción social de los sectores populares. Para el caso de América Latina la economía solidaria tiene tres referentes teóricos centrales: el chileno Luis Razeto (2007) ligado fuertemente al cristianismo ecuménico, el argentino (Coraggio J. L., 2010) cuyas influencias proceden de la llamada corriente substantivista (específicamente de la obra de Karl Polanyi), y finalmente el brasilero Paul Singer (2000) quien abraza una crítica marxista heterodoxa.

\begin{tabular}{lll}
\hline (Razeto, 2007) & (Singer, 2000) & (Coraggio J. L., 2010) \\
\hline La economía solidaria es & La economía solidaria se & La economía solidaria es un \\
incluir la solidaridad en la & prende a valores & proyecto de acción colectiva y \\
economía y no debe & fundamentales del ser & el Estado debe intervenir para \\
depender de los gobiernos & humano y no solo incumbe a \\
de turno & los menos favorecidos & \\
\hline
\end{tabular}

Cuadro Nro. 1: Enfoques de Economía Solidaria Fuente: Elaboración propia

Dentro de este marco conceptual, el término economía social y solidaria tiene dos significados principales complementarios 
i) es un sistema económico cuyo funcionamiento asegura la base material integrada a una sociedad justa y equilibrada o

ii) es un proyecto de acción colectiva (incluyendo prácticas estratégicas de transformación y cotidianas de reproducción) dirigido a contrarrestar las tendencias socialmente negativas del sistema existente, con la perspectiva - actual o potencial de construir un sistema económico alternativo que responda al principio ético ya enunciado.

Finalmente, conviene recordar qué entendemos por "Economía Popular": "En su alcance más amplio, la EP es la economía de los trabajadores, sus familias y comunidades primarias (...) su lógica no es la de la acumulación sin límites sino la de obtención de medios para la reproducción de sus vidas en las mejores condiciones posibles" (Coraggio J. L., 2010).

Entonces, la economía popular y solidaria, genera debate respecto a su conceptualización, es así que varios autores lo definen como:

\begin{tabular}{|c|c|}
\hline AUTOR & CONCEPTO \\
\hline $\begin{array}{l}\text { (Coraggio } \\
\text { J. L., 2010) }\end{array}$ & $\begin{array}{l}\text { Se trata del desarrollo de un modelo alternativo que haga frente a la lógica } \\
\text { capitalista para conseguir el bienestar de todos, teniendo como centro a la } \\
\text { persona y al trabajo valiéndose del mercado para conseguirlo. Además, } \\
\text { incorpora valores como el apoyo mutuo, la cooperación y sobre todo la } \\
\text { solidaridad para conseguir la satisfacción de las necesidades básicas y un } \\
\text { desarrollo para todos y todas. }\end{array}$ \\
\hline $\begin{array}{l}\text { (Herrán, } \\
\text { 2013) }\end{array}$ & $\begin{array}{l}\text { Plantea una definición más funcional: la economía popular y solidaria está } \\
\text { pensada por y para los sectores populares, que a diferencia de los modelos } \\
\text { liberales buscan garantizar la utilización de su propia fuerza de trabajo, como } \\
\text { de los recursos disponibles, la satisfacción de las necesidades básicas a nivel } \\
\text { material e inmaterial. }\end{array}$ \\
\hline $\begin{array}{l}\text { (MIES, } \\
2017)\end{array}$ & $\begin{array}{l}\text { La economía popular y solidaria se reconoció como un nuevo subsistema } \\
\text { económico que mantiene principios basados en la igualdad de oportunidades, } \\
\text { la autogestión, la innovación y la perspectiva de una economía incluyente, que } \\
\text { está en construcción realizando actividades con las economías públicas y } \\
\text { privadas donde su principal objetivo será conformar un único sistema } \\
\text { económico social y solidario. }\end{array}$ \\
\hline $\begin{array}{l}\text { (Muñoz, } \\
\text { 2015) }\end{array}$ & $\begin{array}{l}\text { Considerando el buen vivir de los diversos sectores populares, la Economía } \\
\text { Solidaria se planteó como una alternativa viable a la crisis estructural de } \\
\text { sobreproducción y consumismo; lo que genera que cada vez muchos } \\
\text { ciudadanos no tengan los ingresos necesarios para cubrir necesidades de } \\
\text { consumo. }\end{array}$ \\
\hline $\begin{array}{l}\text { (Saltos \& } \\
\text { Mayorga, } \\
\text { 2004) }\end{array}$ & $\begin{array}{l}\text { La economía popular y solidaria puede definirse como el conjunto de recursos, } \\
\text { capacidades y actividades de las instituciones que reglan la apropiación y } \\
\text { disposición de los recursos en la realización de actividades de producción, } \\
\text { distribución, circulación, financiamiento y consumo, realizadas por los } \\
\text { trabajadores, sus unidades domésticas (familiares y comunales), y las } \\
\text { organizaciones específicas que se dan por extensión para lograr tales fines } \\
\text { (emprendimientos unipersonales y familiares, redes de ayuda mutua, juntas con } \\
\text { fines de gestión económica, cooperativas y asociaciones diversas) organizan } \\
\text { los procesos naturales y las capacidades humanas con el objetivo de reproducir } \\
\text { la vida y fuerza de trabajo en las mejores condiciones posibles }\end{array}$ \\
\hline
\end{tabular}

Cuadro Nro 2: Conceptos de Economía Popular y Solidaria por varios autores Fuente: Elaboración propia 
En el Ecuador, según la Ley Orgánica de Economía Popular y Solidaria y del Sector Financiero Popular y Solidario, los principios en que se deben regir las organizaciones son los siguientes:

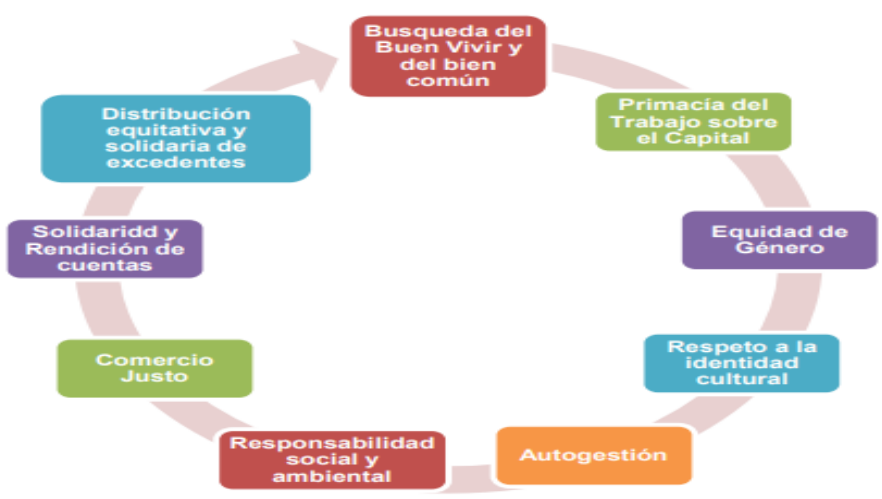

Gráfico Nro. 1: Principios de la Economía Popular y Solidaria Fuente: Ley Orgánica de la Economía Popular y Solidaria y del Sector Financiero popular y Solidario (2011)

En el gráfico siguiente se resumen los principios a los cuales se alinean las organizaciones que conforman el sector económico popular y solidario y que permiten diferenciarlas de las sociedades de capital tradicionales.

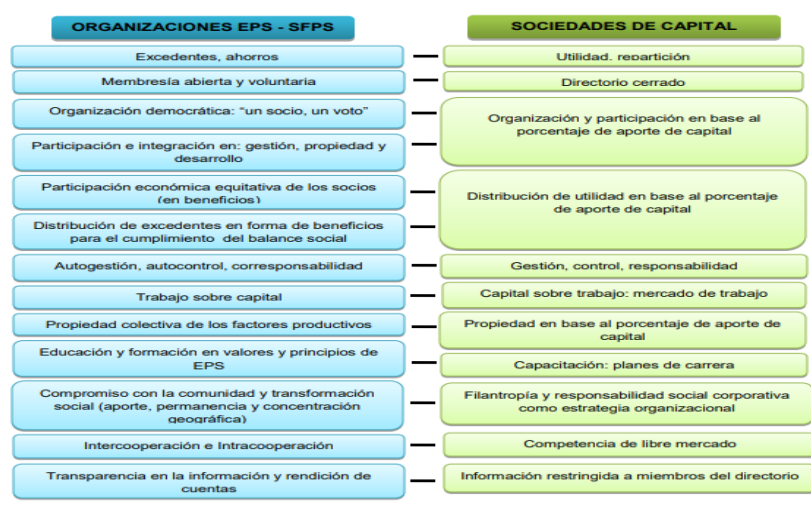

Gráfico Nro. 2: Cuadro comparativo

Fuente: SEPS (2020)

\section{La economía popular y solidaria en Ecuador. -}

Los orígenes y la conformación del movimiento cooperativo ecuatoriano se pueden evidenciar en tres etapas: la primera etapa que inicia aproximadamente en la última década del siglo XIX, cuando se crean en Quito y Guayaquil una serie de organizaciones artesanales y de ayuda mutua, la segunda etapa a partir de 1937 cuando se dicta la primera Ley de Cooperativas con el propósito de dar mayor alcance organizativo a los movimientos campesinos, modernizando su estructura productiva y administrativa mediante la utilización del modelo cooperativista, y la tercera etapa comienza a mediados de los años sesenta con la expedición de la Ley de Reforma Agraria en 1964 que dio lugar a la formación de cooperativas campesinas como forma de integrar los minifundios y 
superación de las formas precarias de tenencia de la tierra y la Segunda Ley de Cooperativas en 1966 que entró para estimular el desarrollo del sector a través de mecanismo como la exención de impuestos, liberación de impuestos a la importación de herramientas y maquinarias agrícolas y semillas; exoneraciones de gravámenes fiscales a las exportaciones de cooperativas artesanales y artísticas y preferencias en las expropiaciones de tierras para organizaciones campesinas. (Jaramillo, 2015).

Con el aparecimiento de la Misión Salesiana y la ayuda de voluntarios italianos inició el establecimiento de Cooperativas de Ahorro y Crédito. Tomando como referencia la jerarquía eclesiástica se creó la Central Ecuatoriana de Servicios Agrícolas (CESA), y el Fondo Ecuatoriano Populorum Progressio (FEPP) con el fin de promocionar y ayudar a los sectores populares. La función de esas instituciones fue precisamente ofrecer un conjunto de servicios (crédito, asistencia técnica y capacitación), que favorecieran el desarrollo de sectores campesinos y urbanos de escasos recursos. La presencia de la Iglesia en gran parte de los casos generaba confianza en los movimientos, inicialmente se pensaba que con su enfoque desinteresado había logrado situarse de manera estratégica en la sociedad civil, en especial en el sector campesino y urbano marginal se presenció un paternalismo que sirvió para integrar a los grupos sociales, sin embargo a largo plazo, tuvo algunos fracasos (Jaramillo, 2015).

El aporte de las ONG al Desarrollo Cooperativo se pueden identificar 3 etapas: la primera con las ONGs de carácter caritativo para atender a los sectores marginados y abandonados; la segunda con la influencia de corrientes ideológicas para apoyar a las actividades de un estado desarrollista; y la tercera con la incorporación de nuevas temáticas de trabajo en el contexto de cooperación internacional y la reducida capacidad de respuesta del Estado en la economía y sociedad, se da entonces una revalorización del funcionamiento y creación de nuevas ONGs en el contexto nacional. (Jaramillo, 2015).

De las experiencias de EPS en el Ecuador, resaltan algunas de gran trascendencia como: CAMARI en 1981, que se responsabiliza del mercadeo a nivel nacional de productos agropecuarios y artesanales que involucra a 140 organizaciones productoras; la Cooperativa de Producción Artesanal "Centros de Bordados Cuenca" desde 1989; la Fundación de Organizaciones Campesinas de Salinas "FUNORSAL" desde 1983 apoyan actividades de los centros agroindustriales, la Unión de Campesinos UCLA desde 1989 con actividades de acopio y comercialización; el Programa Cacao de MCCH desde 1992 con la comercialización de cacao y bordea las 30 organizaciones articuladas.

Por regiones, en la Sierra encontramos a la Unión de Comunas y Cooperativas del Cañar (UPCCC), la Cooperativa CARIACU - Cayambe, la Cooperativa Artesanal - Imbabura, la Unión de Organizaciones Populares de Ayora, Cayambe (UNOPAC), la Unión Interparroquial de Campesinos del Azuay (UNINCA). En la Costa, la Organización de Comunidades Agrícolas de Muisne - Esmeraldas (OCAME), la Unión de Organizaciones Campesinas de Manabí (UOCAM), la Unión de Organizaciones Campesinas de Vinces y Baba (UNOCAVB), la Unión de Organizaciones Campesinas del Litoral (UROCAL), la 
Cooperativa para la comercialización de Esmeraldas. En el Oriente, el Programa de Comercialización PROCOMER, la Coordinadora de la Unión de Campesinos Indígenas para la comercialización (CUCIC), el Programa de Tiendas Comunitarias de Ayuda Mutua (TICAM).

Redes de Economía Popular y Solidaria que se destacan son la Fundación Consorcio de Queserías Rurales Comunitarias ubicadas en seis provincias de la Sierra, la RENAFIPSE organización de integración de cooperativas de ahorro y crédito, cajas de ahorro y crédito y bancos comunales en Ecuador; y ONG's como: Fundación de Organizaciones Campesinas de Salinas, Fondo Ecuatoriano Populorum Progressio, Promoción Humana Diocesana de Guaranda y Fundación Misión Salesiana (Jaramillo, 2015).

Por otro lado, es usual utilizar la denominación de Economía Solidaria para la promoción de actividades económicas asociativas auto gestionadas por los propios trabajadores a fin de integrarse al sistema económico que los excluyó. Por agregación y encadenamientos, los emprendimientos populares y solidarios pueden ir constituyendo un sector orgánico de peso creciente en la economía, capaz de moderar los efectos de la insuficiencia dinámica del modelo productivo y disputar la hegemonía de las formas capitalistas, su paradigma empresarial y sus valores. (Askunze, 2007).

En Ecuador, a partir de 2008 legalmente existen tres tipos de economía: la primera es la pública, que parte del accionar del Estado con sus instituciones y servicios; la segunda es la privada, formada por empresas grandes, medianas y pequeñas cuya finalidad principal es la obtención de lucro; y la tercera es la economía popular y solidaria, representada por aquellos actores sociales informales que realizan un sin número de actividades económicas populares y que contribuyen significativamente a la economía del país. Todas representadas en la siguiente figura:

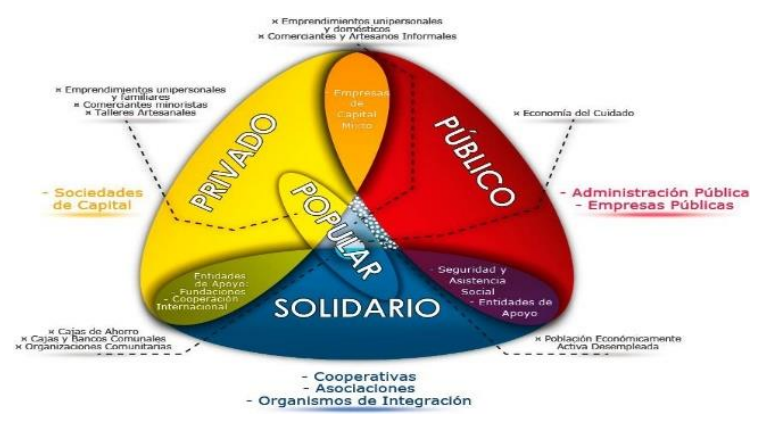

Figura 1: Sectores de la Economía Popular y Solidaria Fuente: SEPS, (2020)

El Ecuador ha dirigido su economía a este tercer sector, tratando de dotarlo de factores de producción y construyendo relaciones en producción, distribución, consumo y financiación, basadas en principios fundamentales, valores y características que sustentan esta actividad, como son:

a. La búsqueda de la satisfacción en común de las necesidades de sus integrantes, especialmente las básicas de autoempleo y subsistencia. 
b. Su compromiso con la comunidad, el desarrollo territorial y la naturaleza.

c. La ausencia de fin de lucro en la relación con sus miembros.

d. La no discriminación, ni concesión de privilegios a ninguno de sus miembros.

e. La autogestión democrática y participativa, el autocontrol y la auto responsabilidad.

f. La prevalencia del trabajo sobre el capital, de los intereses colectivos por encima de los individuales y de las relaciones de reciprocidad y cooperación, sobre el egoísmo y la competencia (ASAMBLEA NACIONAL, 2011) .

Con respecto a los valores, prevalecen la justicia, honestidad, transparencia y responsabilidad social y, los principios se sustentan en la ayuda mutua, el esfuerzo propio, la gestión democrática, el comercio justo y el consumo ético. (ASAMBLEA NACIONAL, 2011).

En cuanto a la Institucionalidad de la EPS en el Ecuador existen avances significativos en temas de rectoría, regulación, control, promoción; que procura garantizar el correcto desenvolvimiento de este sector, como se puede observar en la tabla 1.

\begin{tabular}{|c|c|c|c|}
\hline RECTORIA & REGULACION & CONTROL & FOMENTO \\
\hline $\begin{array}{l}\text { Comité Interinstitucional } \\
\text { MCDS-MCPEC-MCPE }\end{array}$ & $\begin{array}{l}\text { SECTOR NO } \\
\text { FINANCIERO: MCDS }\end{array}$ & & ón: IEPS \\
\hline $\begin{array}{l}\text { Junta de política y } \\
\text { regulación monetaria y } \\
\text { financiera }\end{array}$ & $\begin{array}{l}\text { Junta de Política y } \\
\text { regulación Monetaria y } \\
\text { Financiera } \\
\text { REGULACIONES } \\
\text { SECTORIALES: Agencia }\end{array}$ & $\begin{array}{l}\text { Superintendenci } \\
\text { a de Economía } \\
\text { Popular y } \\
\text { Solidaria }\end{array}$ & $\begin{array}{l}\text { Financiamiento. } \\
\text { CONAFIPS } \\
\text { Corporación de }\end{array}$ \\
\hline $\begin{array}{l}\text { Asesoría: Consejo } \\
\text { Consultivo (participación } \\
\text { ciudadana) }\end{array}$ & $\begin{array}{l}\text { Nacional de Tránsito. } \\
\text { Ministerio de Desarrollo } \\
\text { Urbano y Vivienda }\end{array}$ & & $\begin{array}{l}\text { depósitos, Fondo de } \\
\text { liquidez y Fondo de } \\
\text { Seguros Privados }\end{array}$ \\
\hline
\end{tabular}

Tabla 1: Institucionalidad de la economía popular y solidaria

Fuente: Ley Orgánica de Economía Popular y Solidaria LOEPS (2011)

La Superintendencia de Economía Popular y Solidaria (SEPS) es una institución de supervisión y control y entre sus principales responsabilidades son: control y regulación de OEPS, conocer y aprobar las reformas a los estatutos de las OEPS, registrar nombramientos de directivos y representantes legales de las OEPS, proponer regulaciones y normativas, auditoría de las OEPS.

El Instituto de Economía Popular y Solidaria IEPS es quien ejecuta la política pública, coordina, organiza y aplica los planes, programas y proyectos que contribuyen a la construcción del Sistema Económico Social y Solidario y del Sumak Kawsay o Buen vivir (ASAMBLEA, 2011). Los ejes estratégicos que actualmente maneja el IEPS se enmarca en el fortalecimiento organizativo, el fomento productivo, intercambio y mercados (IEPS, 2019). 
La SEPS (2020) registra que existen 12.443 asociaciones, 2588 cooperativas y 50 organizaciones comunitarias dentro del sector de la economía popular y solidaria No financiera.

\section{Metodologia.}

La investigación es de naturaleza descriptiva, transversal y correlacional. a) Descriptiva debido a que el objetivo es caracterizar el contexto y analizar las organizaciones de economía popular y solidaria en el caso del cantón Cuenca, b) Transversal ya que los datos se van a recolectar a través de un solo instrumento administrado en un único momento en el tiempo, y c) Correlacional ya que se utilizará técnicas estadísticas para medir la fuerza de la relación entre las variables ingreso mensual y número de socios/as de organizaciones de economía popular y solidaria (Hernández, Fernández, \& Batista, 1991).

El cuestionario utilizado es una herramienta de la Guía metodológica para el mapeo y relevamiento de la economía popular y solidaria en Latinoamérica y Caribe (Coraggio, Arancibia, \& Deux, 2010).

Para este caso se utilizará un muestreo estratificado que permitirá el calculó de una muestra. El tamaño de la población total (universo) se obtiene del total de población que se encuentra en el sector no financiero de la economía popular y solidaria en el cantón Cuenca, tomado como fuente los datos de la Superintendencia de Economía Popular y Solidaria, como entidad de control que tiene los datos actualizados a Enero del 2021; a partir de estos datos y con un nivel de confianza del 90\%, error máximo admisible del $10 \%$ y un valor de la proporción de población que representa el fenómeno constante de 0.5 , debido a que no se conocía el mismo se calculará la muestra.

Finalmente, se analiza 2 variables más representativas como son el número de socios/as y el ingreso mensual de la organización, para lo cual se usará el SPSS que es un formato que ofrece IBM para un análisis completo utilizado para realizar la captura y análisis de datos para crear tablas y gráficas con data compleja.

\section{Población y muestra}

Según la SEPS (2020), en el Ecuador existen 12.443 asociaciones, 2588 cooperativas y 50 organizaciones comunitarias dentro del sector No financiero de la economía popular y solidaria. En Azuay existen 524 organizaciones del sector no financiero de la economía popular y solidaria y en el caso del cantón Cuenca la población en estudio es de 266 organizaciones en estado activo: el 57\% son asociaciones (es decir 151), el $42 \%$ son cooperativas (es decir 113) y el $1 \%$ son uniones (es decir 2). 
La muestra fue obtenida mediante un método probabilístico, y el cálculo se hizo utilizando la varianza máxima (p x q a un valor máximo de 0,5 ), con un nivel de confianza de $90 \%$ y un error máximo admisible de $10 \%$. Estos valores fueron aplicados en la fórmula de Aguilar (2005), para el cálculo de una muestra representativa para poblaciones finitas. Para la selección de las organizaciones se utilizó un rnuestreo aleatorio simple, en el que tanto las asociaciones, cooperativas y uniones tienen la misma oportunidad de ser seleccionados. (Lind, Marchal, \& Wathen, 2012). La muestra del presente estudio quedó constituida por un total de 54 organizaciones de economía popular y solidaria sector no financiero, que al momento de aplicar encuestas se contó con la respuesta de 59 organizaciones, con lo cual se realizó los análisis respectivos.

\section{Instrumento de recolección de datos}

La investigación se llevó a cabo por medio del método de encuesta, y el instrumento de recolección de datos fue una encuesta semi-estructurada, que combina preguntas de respuesta con opciones cerradas, de respuesta única, múltiple, y de respuestas abiertas, que constó de 54 ítems, dentro de la Guía para mapeo y de relevamiento de la economía popular y solidario en Latinoamérica y Caribe (Coraggio, Arancibia, \& Deux, 2010).

Este instrumento, se encuentra organizado en función de tres ejes temáticos: Articulación de los actores de la economía popular solidaria y la economía social y solidaria; Características de las organizaciones de economía popular solidaria (OEPS); y Características de los productos y servicios de las organizaciones de economía popular y solidaria (OEPS).

La fiabilidad del cuestionario se determinó mediante el análisis de la consistencia interna que hace referencia al grado de relación que muestren los ítems que componen el instrumento (Oviedo \& Campo-Arias, 2005). Con este propósito se usó el coeficiente de Alfa de Cronbach, cuyo valor obtenido en el análisis fue de 0.881; significa que el cuestionario tiene fiabilidad (Kline, 1998).

Para la recolección de la información, se estructuró la encuesta y se aplicó vía online mediante la herramienta formularios de Google. Mediante email personal se envió el link de acceso al formulario a emails de organizaciones, y se facilitó instrucciones de aplicación.

Para la selección de la muestra, sistematización, análisis y presentación de resultados, se usó instrumentos como: Microsoft Excel 2010 para generar números aleatorios (random) que permitan seleccionar al azar a los participantes. El SPSS 19.0, para la sistematización y obtención de estadísticos para el análisis descriptivo de resultados, de fiabilidad del instrumento, y de correlación entre variables que permita identificar la relación entre el número de socios (hombres y mujeres) y el ingreso mensual de la organización.

\section{Resultados.}


Para la presentación e interpretación de los resultados se realizó dos tipos de análisis uno descriptivo y otro correlacional. Se analiza las teorías sobre la economía popular y solidaria en el desarrollo local, específicamente el sector NO financiero. Se caracteriza las organizaciones de la economía popular y solidaria del sector No financiero en el caso del Cantón Cuenca. Finalmente, se establece la relación entre las variables ingresos económicos mensuales y número de socios y socias.

\section{Análisis descriptivo.}

Es un método que permite organizar, resumir y presentar datos de manera informativa fáciles de interpretar (Lind, Marchal, \& Wathen, 2012). Por ello, para los fines de la presentación de los resultados en este estudio, los datos fueron procesados de manera descriptiva.

Las organizaciones de economía popular y solidaria -OEPS son aquellas organizaciones socioeconómicas asociativas, constituidas por un grupo de personas pertenecientes a diferentes hogares que se agrupan para compartir recursos, desarrollar procesos de trabajo conjunto, abastecerse de insumos, producir o comercializar colectivamente servicios o bienes de forma autogestionaria, orientadas al intercambio en el mercado u otras formas de comercio como el comercio justo, trueque (Coraggio, Arancibia, \& Deux, 2010)

De acuerdo a datos de la SEPS (2020) en el Cantón Cuenca existen 308 organizaciones del sector NO financiero de economía popular y solidaria (OEPS), el $86 \%$ en estado "activo" es decir 266 OEPS, el 11\% en estado "liquidadas" es decir 33 OEPS y el 3\% en estado "en liquidación" es decir 9 OEPS.

La investigación se ejecuta en base a las organizaciones de EPS que están en estado activo en Cantón Cuenca, identificadas de la siguiente manera: 151 Asociaciones que corresponde al 57\%, 113 Cooperativas que corresponde al $42 \%$ y 2 Uniones, es decir el $1 \%$.

De acuerdo a la base de datos y registro de OEPS (SEPS, 2020), el 65\% de las organizaciones de EPS se ubican en las parroquias urbanas del cantón Cuenca, que brindan servicios de transporte, limpieza, alimentación y productos textiles, agropecuarias; el 35\% de las OEPS se ubican en las parroquias rurales del Cantón Cuenca que ofertan productos agropecuarias, textiles y servicios de alimentación.

\section{Articulación de los actores de la Economía Popular Solidaria y la Economía Social y Solidaria}

En base a la aplicación de encuestas, se identifica que sólo el 25\% de las organizaciones de economía popular y solidaria son parte de organizaciones de integración, como son: Red Agroecológica del Austro -RAE, Asociación de Productores del Austro - APA, Red de Economía Solidaria del Austro - REDESOL, a las cuales están vinculados organizaciones agropecuarias o agrícolas; por otro lado, organizaciones textiles, limpieza, mantenimiento están vinculadas a Cámara de la EPS del Azuay y Cañar. Esta articulación 
en redes u organismos de integración les permiten a las OEPS acceder en forma conjunta a procesos de capacitación, compra pública, articulación con entidades públicas, acceso a información y formación tecnológica (estrictamente técnica o social), uso de espacios para comercialización. Las OEPS son legalizadas y cumplen con todos los requisitos exigidos para su funcionamiento y actividad productiva, como son: RUC, RUP, permisos de funcionamiento, patente, permiso de bomberos, permisos de salud, registro ARCSA, dado que proveen a través de procesos de compra pública por lo que cumplen con requisitos y exigencias de las entidades a las cuales proveen.

La ley de economía popular y solidaria fue aprobada por la Asamblea (2011), por tanto, la Superintendencia estableció las normativas para que las organizaciones puedan adecuar sus estatutos, además las instituciones como Ministerio de Agricultura y Ganadería MAGAP, Ministerio de Industrias y Productividad -MIPRO, Ministerio del Ambiente MAE, Ministerio de Inclusión Económica y Social -MIES, migren sus bases de datos a las SEPS. Este proceso generó que desde el año 2013 se cuente con la nueva base de datos de OEPS en el país, y en el caso del Cantón Cuenca se obtiene el siguiente registro:

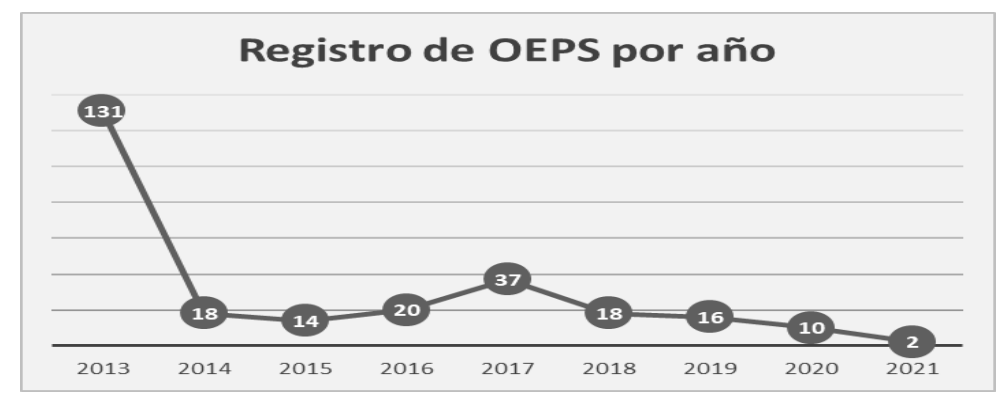

\section{Gráfico Nro. 5: Registro de OEPS en Cantón Cuenca por año Fuente: Elaboración propia con datos SEPS, 2021}

El uso de espacio para sus actividades productivas es determinante para las organizaciones, el 58\% realizan sus actividades desde su domicilio como son las organizaciones agropecuarias, agrícolas, textiles; el 15\% usa espacios alquilados como son las organizaciones de limpieza y mantenimiento, transporte; el $12 \%$ el local es prestado, el $8 \%$ lo usan como parte de proyectos o concesionado, el $5 \%$ ocupado de hecho y solamente el $2 \%$ es local propio. Respecto a la maquinaria y equipos el $61 \%$ es de cada socio, el $29 \%$ es de la organización, el $7 \%$ no tiene, y el $3 \%$ es prestado. Debido a esta situación las OEPS no hacen inversiones en locales, lo realizan en sus equipos o maquinarias que les permiten mejorar su nivel de productividad. (vehículos para transporte, equipos de limpieza, equipos de cocina, equipos para postcosecha), y sobre todo prestar el servicio a la entidad pública o privada que los contrata.

El uso de tecnología es una herramienta fundamental, por lo que el 100\% tiene acceso a computadoras, impresoras y equipos que les permita enviar oficios, propuestas y les permita realizar gestiones para sus actividades productivas. El acceso a redes sociales al 
ser gratuito les permite acceder para promocionar sus actividades, productos, bienes o servicios, pero solamente el $20 \%$ usa redes como Facebook, Twitter, Instagram; las mismas que están relacionadas al mercado de sus productos, bienes o servicios, ya que el $85 \%$ lo comercializa a nivel cantonal, el $12 \%$ a nivel provincial, el $2 \%$ a nivel de la región 6 (Azuay, Cañar y Morona S.), y tan solo el 1\% comercializa a nivel internacional (Coop. Centro de Bordados Cuenca), que exporta sus productos a EEUU. y Europa.

\section{Características de las Organizaciones de Economía Popular Solidaria (OEPS)}

En base a los instrumentos aplicados se identifican las características de las organizaciones de acuerdo a su tipo, grupo y clase.

En las Asociaciones de EPS, la clase de organizaciones con mayor concentración son: agropecuarias (33\%), textiles (25\%), alimentación (27\%), limpieza (23\%), y otros servicios $(11 \%)$, el resto en menor proporción.

\begin{tabular}{|l|r|}
\hline \multicolumn{2}{|l|}{ Clase Organización } \\
\hline Grupo Producción
\end{tabular}

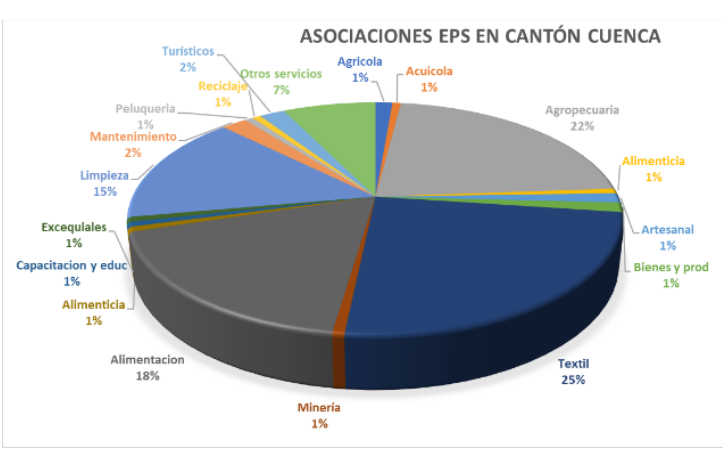

Gráfico Nro. 3: Asociaciones EPS en Cantón Cuenca

Fuente: Elaboración propia con datos SEPS, 2021

En las Cooperativas de EPS, la clase de organizaciones con mayor concentración son: transporte $(89 \%)$, agropecuaria (8\%), otros servicios $(5 \%)$, el resto en menor proporción, como se puede ver en la tabla: 


\begin{tabular}{|c|c|}
\hline Clase Organización & Nro OEPS \\
\hline \multicolumn{2}{|l|}{ Grupo Consumo } \\
\hline Bienes y productos & 3 \\
\hline \multicolumn{2}{|l|}{ Grupo Vivienda } \\
\hline Adquisición & 1 \\
\hline Construcción & 2 \\
\hline \multicolumn{2}{|l|}{ Grupo Producción } \\
\hline Agropecuaria & 8 \\
\hline Textil & 1 \\
\hline Artesanal & 1 \\
\hline \multicolumn{2}{|l|}{ Grupo Servicios } \\
\hline Capacitación & 1 \\
\hline Educación & 1 \\
\hline Otros & 5 \\
\hline Turismo & 1 \\
\hline Transporte & 89 \\
\hline Total & 113 \\
\hline
\end{tabular}

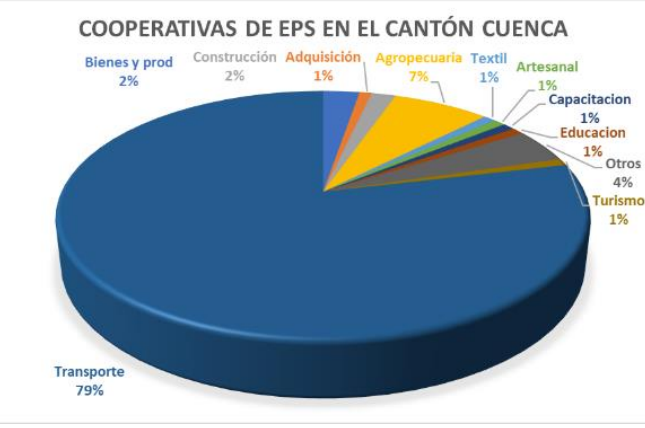

Gráfico Nro. 4: Cooperativas de EPS en el cantón Cuenca

Fuente: Elaboración propia con datos SEPS, 2021

Tabla Nro 3: Cooperativas de EPS en el cantón Cuenca

Fuente: Elaboración propia con datos SEPS, 2021

En las Uniones de EPS, la clase de organizaciones se concentran en servicios de transporte $(100 \%)$.

\begin{tabular}{lllll}
\hline Tipo Organización & \multicolumn{3}{l}{ Nro $\%$} \\
& Grupo & Clase Organización & \multicolumn{2}{l}{ OEPS } \\
\hline Unión & Servicios & No específica & 2 & $100 \%$ \\
& & Total & 2 & $100 \%$ \\
\hline
\end{tabular}

Tabla Nro 4: Clasificación de Uniones de EPS en el cantón Cuenca Fuente: Elaboración propia con datos SEPS, 2021

$\mathrm{Al}$ analizar la ubicación geográfica de las organizaciones de EPS se identifica que el $65 \%$ se encuentran en el área urbana (174 OEPS) y el 35\% en el área rural (92 OEPS). El 87\% de las OEPS comercializan lo que producen, sean bienes, productos o servicios, mientras que el $13 \%$ es para autoconsumo. Los clientes o consumidores, como el sector público demandan productos, bienes o servicios como: mantenimiento, limpieza, textiles, alimentación que acceden mediante procesos de contratación pública en el cual participan el $81 \%$ de las OEPS, pero también el 59\% de las OEPS comercializan con sectores privados. No se identifica que las OEPS generen producción para intercambio o trueque. Por otro lado, los proveedores de las organizaciones en el 100\% son sectores privados, emprendimientos particulares, en algunos casos los propios socios/as y no están enlazados con otras organizaciones de EPS. El alcance de las organizaciones está determinado con su ámbito de producción y comercialización: el 85\% de la OEPS a nivel cantonal, el 12\% a nivel provincial, el 2\% a nivel de la región 6 (Azuay, Cañar y Morona S.), y tan solo el $1 \%$ comercializa a nivel internacional (Coop. Centro de Bordados Cuenca), que exporta sus productos a EEUU. y Europa. 
En base a estos elementos, se establece las siguientes características en las organizaciones de economía popular y solidaria (OEPS):

- Las OEPS productores de bienes son aquellos que se dedican a la transformación de materias primas (por ejemplo, frutas, hortalizas, madera, textiles, etc.) en productos elaborados, a partir de procesos de manufactura artesanal o industrial, que se destinan tanto al consumo final (pan, muebles de madera, viviendas, vestido, etc.) o como insumos de otros procesos de transformación (frutas procesadas, ladrillos, envases, textiles, etc.). En esta categoría se identifican aquellas OEPS con bajas escala de productividad y complejidad, escaso uso de maquinarias y en general de "bajo" desarrollo tecnológico.

- Las OEPS que prestan servicios al público en general sean de uso individual o colectivo, y referidos tanto a necesidades personales y/o familiares, como son: servicios turísticos, cuidado de niños, transporte de carga o de pasajeros, alimentación, etc., como a requerimientos de las actividades productivas de la economía popular: empaque y/o comercialización de productos, limpieza de locales, servicios de mantenimiento y limpieza. Estos servicios pueden ser prestados a clientes externos a la organización, a través del cobro de un precio (tarifa, tasa, arancel, etc.), como a sus propios miembros (a través de cuotas de socios u otros aportes a la organización, etc.). En esta categoría las organizaciones proveen al Estado servicios como: alimentación, limpieza, mantenimiento a entidades como Centros Infantiles del Buen Vivir -CIBVs del MIES, Ministerio de Salud, Ministerio de Educación, Empresas Públicas a través de procesos de compra pública.

- Las organizaciones que prestan servicios de comercialización son aquellos que intermedian entre productores y consumidores, ofreciendo a sus socios o clientes servicios como: creación y organización de espacios de intercambio o trueque, organización logística y distribución de los productos, búsqueda y/o generación de nuevos mercados, obtención de precios ventajosos, entre otros. Algunos OEPS asociados a esta categoría son las organizaciones de comercio justo, las redes de comercialización alternativa, de consumo responsable, las redes de trueque, etc.

Las organizaciones de EPS de acuerdo a la información levantada, se constituyen con el fin de solucionar problemas en común en un grupo de personas, como es el acceso a recursos económicos, por lo que las OEPS tratan de generar actividades económicas en las cuales se beneficien todos los socios/as, y en algunos casos generan empleo adicional pudiendo contratar trabajadores.

De acuerdo al análisis realizado, se identifica que las organizaciones agropecuarias, conformadas por productores/as, producen en sus terrenos familiares, alquilados o concesionados, y juntan la cosecha para comercializar, pero el ingreso económico no es contabilizado a través de las organizaciones, este ingresa de forma individual al bolsillo del productor, por tanto la OEPS no declara ingresos, es decir no factura como organización provocando un subregistro contable real de ingresos y egresos. En el caso 
de las organizaciones de transporte (camionetas, taxis) sucede algo similar, cada persona se encarga de sus ingresos y gastos dado que usan unidades móviles y no a través de la organización, y al final del mes solo hacen un aporte establecido en asamblea entre socios/as. En el caso de organizaciones que prestan servicios de alimentación, mantenimiento, limpieza, o proveen textiles a través de procesos de compra pública, la organización es la que factura, tributa y al final realiza compensación económica a sus socios/as de acuerdo al trabajo realizado o realiza el pago a trabajadores si ha contratado, pudiendo tener datos productivos; salvo el caso de las organizaciones textiles que sus socios/as realizan actividades productivas paralelas ya que tienen sus propios talleres y con personal contratado.

Las organizaciones luego de cubrir sus gastos, tributación y gastos generados del proceso administrativo, dan ciertos beneficios adicionales a sus socios/as, como son: ofertan asistencia técnica, capacitación, alimentación, transporte, servicios de salud y apoyo interno. Al ser organizaciones de EPS estas no tienen relación de dependencia por que realizan actos solidarios (ASAMBLEA NACIONAL, 2011), no así los trabajadores contratados que acceden a todos los beneficios de ley.

A pesar de lo establecido en la LOEPS (2011), muchos socios/as de las organizaciones de EPS acceden a diferentes tipos de seguro, como son: en el 25\% de las OEPS los socios/as acceden al seguro campesino (organizaciones agropecuarias), en el 75\% de las OEPS los socios/as acceden al seguro voluntario (son socios/as de organizaciones y a la vez son patrones en sus talleres -textiles- por tanto tiene personal bajo dependencia), y en $8 \%$ de las OEPS sus socios/as mantienen seguro como trabajo no remunerado del hogar.

Respecto al origen de los recursos es importante señalar que los socios/as gestionan los mismos a través de diversas fuentes, como son: sus propios aportes o recursos en un $88 \%$, a través de bancos privados o públicos el $49 \%$, y a través de sector Cooperativo el $72 \%$. El acceso a través del sistema financiero les permite cubrir sus necesidades, pero también les da solvencia económica y garantía financiera para acceder a préstamos o crédito, para garantía en contratos con sector público.

Por otro lado, la edad promedio de los socios/as oscila entre 30 y 65 años, por lo que es población económicamente activa que ejecuta actividades productivas constantemente. Debido a la normativa establecida en la LOEPS (2011) el mínimo de personas para constituir una organización es de 13 personas, al analizar el promedio de integrantes de las organizaciones oscila por los 18 socios/as. De la muestra analizada, es decir de 59 organizaciones de EPS se identificó 1098 socios/as, constituidas por $69 \%$ mujeres y $31 \%$ hombres. La etnia predominante es la mestiza. Estas organizaciones no están constituidas por población en situación de riesgo como son: ex presidiarios, adictos, en situación de calle, con discapacidad.

En cuanto a los ingresos percibidos, es una deficiencia del $90 \%$ de las organizaciones analizadas que, al no tener contabilidad única de la OEPS, donde se registre ingresos y 
gastos, no se evidencia si tienen rendimientos económicos relevantes y solo se registra cuando hay procesos de contratación pública, como es el caso de organizaciones de limpieza, mantenimiento, alimentación, textil. Debido a las normativas de la LOEPS, los socios/as realizan actos solidarios por lo que sus compensaciones económicas las realizan en función del trabajo realizado en el 50\% de las OEPS que tienen contratos más frecuentes con el sector público. Las contrataciones de personal son esporádicas, en función de contratos que tienen con el sector público o privado, lo que permite contratar ocasionalmente al mes, bimensual o trimestral; en su mayoría las actividades realizan los socios/as. En el caso de contar con excedentes al final del año, la misma es usada de acuerdo a la normativa de la SEPS, es decir 5\% pago de tributos a SEPS, $45 \%$ repartición entre socios/as, $50 \%$ capitalización de la organización.

\section{Características de los productos y servicios de las OEPS}

Las características de los bienes, productos y servicios de las organizaciones de economía popular y solidaria identificados en el Cantón Cuenca, son:

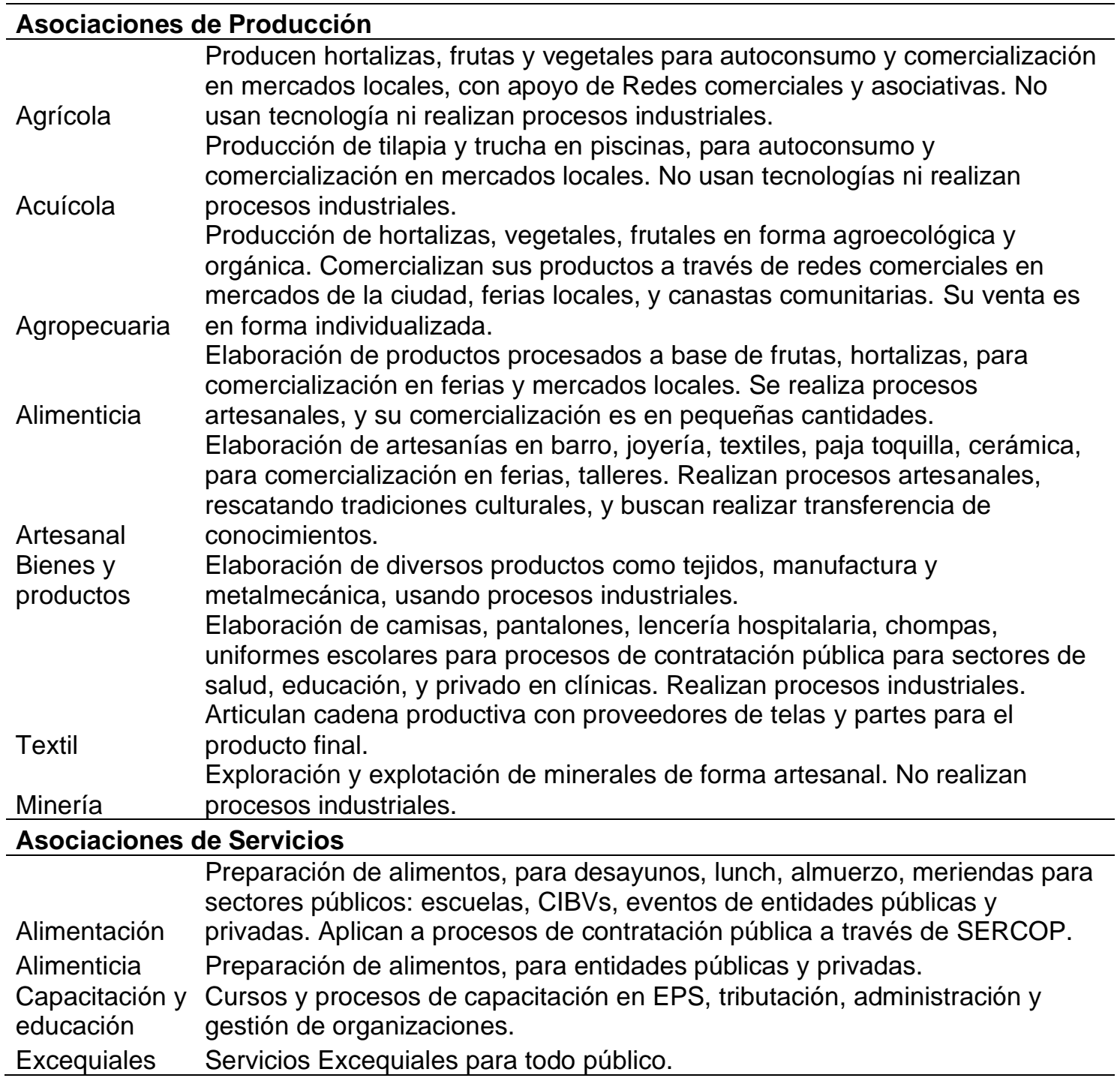


Servicios de limpieza de oficinas, edificios de entidades públicas o privadas, a través de procesos de contratación pública. Usan equipos y maquinaria especializada para limpieza. Algunas OEPS elaboran y comercializan sus

Limpieza propios productos de limpieza para desinfección, jabones, shampoo.

Mantenimiento electricidad. Para entidades públicas y privadas.

Servicios de belleza, corte de cabello y peinados para hombres, mujeres, niños/as. Se juntan con la finalidad de acceder a procesos de capacitación,

Peluquería calificación artesanal.

Realizan reciclaje de materiales como cartón, plástico, vidrio. Lo venden a recicladoras más grandes diariamente, en forma individual. Se asocian para beneficiarse de apoyo institucionales a sus familiares e hijo/as, al estar

Reciclaje considerados como población en situación de vulnerabilidad.

Turísticos organizaciones, como áreas en sus zonas de influencia.

Tabla Nro 5: Características de las Asociaciones de EPS en el cantón Cuenca Fuente: Elaboración propia

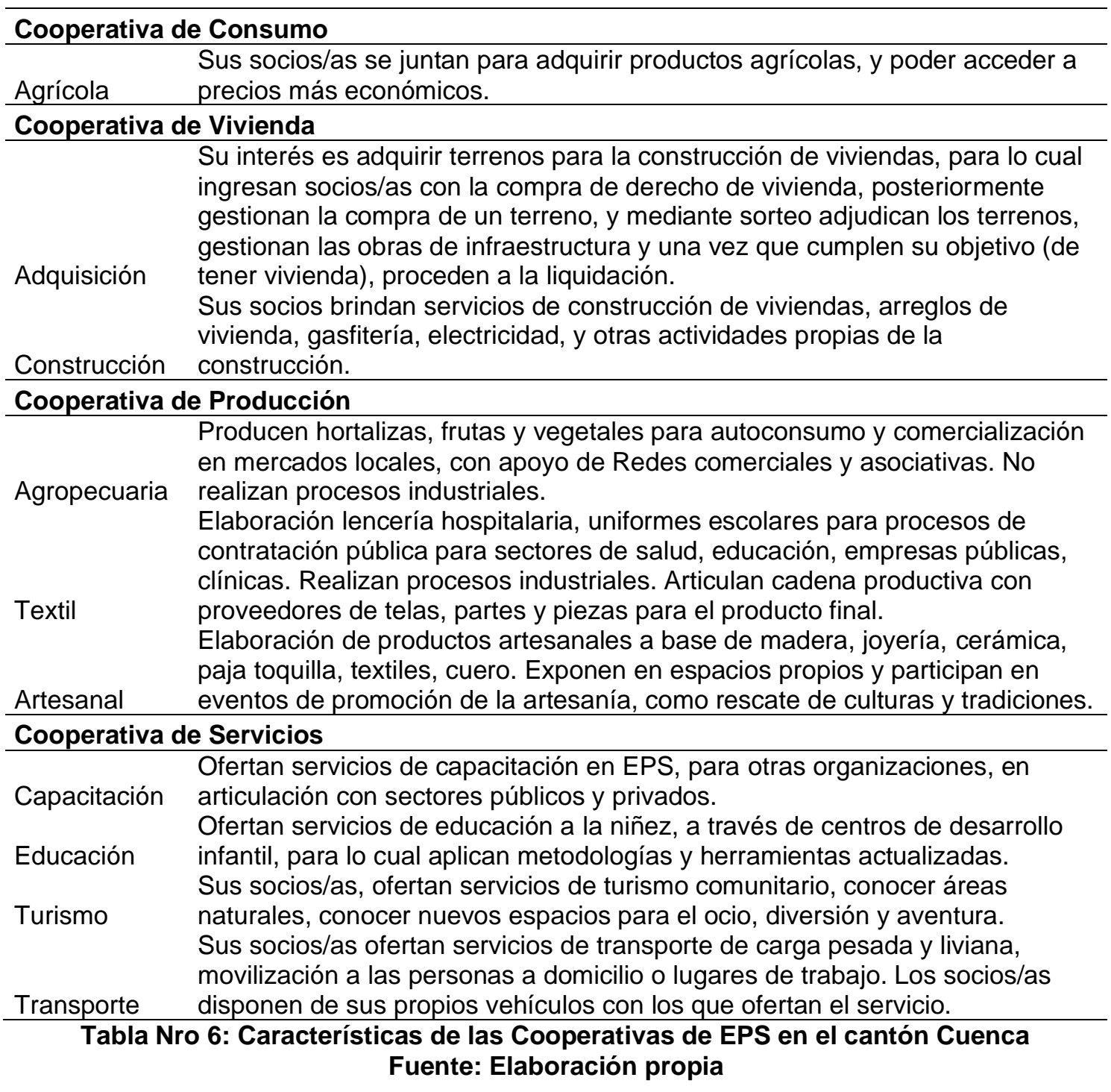

Uniones 
Ofertan servicios de transporte de carga pesada y liviana, movilización a las personas a domicilio o lugares de trabajo. Los socios/as disponen de sus

Servicios propios vehículos con los que ofertan el servicio.

Tabla Nro 7: Características de las Uniones de EPS en el cantón Cuenca

Fuente: Elaboración propia

\section{Análisis de correlaciones}

El análisis de correlación tiene como objetivo reportar la asociación o fuerza de relación entre dos variables aleatorias. Para poder cuantificar este grado de variación conjunta, es necesario determinar un coeficiente de correlación, que puede ser el de Pearson, el de Tau-b de Kendall y el de Spearman, para el caso de correlaciones bivariados. Los valores posibles del coeficiente tienen rango de $-1 \mathrm{a}+1$, los valores de $-1.0 \mathrm{y}+1.0$, indica relación perfecta o significativa, y el valor de cero muestra la correlación nula.

En el presente estudio se determinó el coeficiente de correlación producto-momento, más conocido como $\mathrm{r}$ de Pearson, para obtener una medida de la fuerza de la relación por dimensiones y una general entre número de socios (hombres y mujeres) y el ingreso mensual de la organización. Se consideró el criterio de Guilford, quien propone la siguiente interpretación descriptiva:

\section{Positiva}

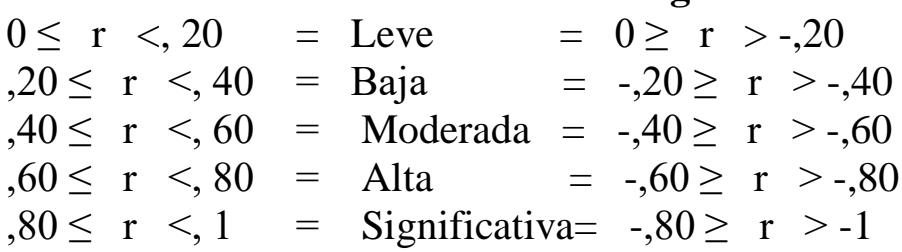

Mediante el uso del programa estadístico SPSS, se pudo emparejar las dimensiones del número de socios (hombres y mujeres) y el ingreso mensual de las organizaciones de economía popular y solidaria, sector NO financiero, obteniendo las correlaciones que se detallan en la Tabla 7.

Tabla Nro. 7: Análisis de correlación

\begin{tabular}{|c|c|c|c|c|}
\hline & $\begin{array}{l}\text { Cuantos } \\
\text { socios/as } \\
\text { conforman su } \\
\text { organización? }\end{array}$ & $\begin{array}{l}\text { Cuantos de } \\
\text { socios/as } \\
\text { son } \\
\text { mujeres? }\end{array}$ & $\begin{array}{l}\text { Cuantos } \\
\text { socios/as } \\
\text { son } \\
\text { hombres? }\end{array}$ & $\begin{array}{l}\text { ¿Cuál el monto de } \\
\text { ingresos de } \\
\text { recursos que recibe } \\
\text { la organización al } \\
\text { mes por venta o } \\
\text { comercialización de } \\
\text { sus productos, } \\
\text { bienes o servicios? }\end{array}$ \\
\hline $\begin{array}{l}\text { Cuantos socios/as } \\
\text { conforman su } \\
\text { organización? }\end{array}$ & 1 & ,800** & ,711 &, $906^{* \star}$ \\
\hline $\begin{array}{l}\text { Cuantos de socios/as } \\
\text { son mujeres? }\end{array}$ & ,800** & 1 &, 146 &, $762^{\star *}$ \\
\hline
\end{tabular}


Cuantos socios/as son hombres?

¿Cuál el monto de

, $711^{* *}$

,146

$600^{* *}$

ingresos de recursos

, $906^{* *}$

, $762^{\star \star}$

, $600^{* *}$

1

que recibe la

organización al mes por

venta o comercialización

de sus productos,

bienes o servicios?

\section{${ }^{\star \star}$ La correlación es significativa al nivel 0,01 (bilateral)}

Finalmente, el estudio demuestra que existe una relación positiva moderada que es significativa al nivel de $\mathrm{p} p<0,01$, entre número de socios/as y el ingreso mensual, sin llegar a establecer un sentido de causalidad.

\section{Conclusiones.}

- Al analizar las teorías de la economía popular y solidaria, identificamos que la EPS es el vínculo de: recursos, capacidades y actividades e instituciones que tienen por objeto regularizar, en base a principios de solidaridad, el manejo de estos recursos para el desarrollo de actividades en procesos de producción, distribución, circulación, financiamiento y consumo que se encuentren organizadas por los propios trabajadores y sus familias, a través de organizaciones comunitarias o asociatividad auto gestionada.

- Las actividades productivas de las OEPS en el cantón Cuenca son diversas, enfocadas en los últimos años en cubrir la oferta y demanda del sector público, y con el apoyo de instituciones como SRI, IESS, SERCOP, IEPS, SECAP, UNIVERSIDADES han accedido a procesos de formación y capacitación, que les permita mejorar sus procesos productivos y administrativos, para incrementar su productividad y competividad, sin embargo los procesos burocráticos de la compra pública ha generado muchos problemas socioeconómicos en las organizaciones, poniendo en riesgo la sostenibilidad de las OEPS, ya que sus miembros por la falta de ingresos económicos han tenido que buscar fuentes de ingresos, sea por empleo o actividades laborales en forma individualizada.

- Las OEPS son una alternativa de desarrollo económico local, ya que el trabajo asociativo ha permitido fortalecer los lazos internos de los grupos en situación de vulnerabilidad y riegos como son: mujeres, mujeres en situación de violencia, que han tenido en las organizaciones una oportunidad de trabajar en conjunto y acceder a capacitación y asistencia técnica.

- Los principios y valores con los cuales se rigen las OEPS permite resistir la exclusión social al que estaban expuestos las personas (hombres y mujeres), y que han visto una opción de igualdad y equidad, acceso a recursos, buen trato, mejora de su condición económica.

- El estudio demuestra que existe una relación positiva moderada que es significativa al nivel de $\mathrm{p}$ p $<0,01$, entre número de socios/as y el ingreso mensual, 
sin llegar a establecer un sentido de causalidad lo que permite concluir que, si mayor es el número de socios/as en una organización, permite mejorar el ingreso económico. Al tener más socios/as pueden acceder a más contratos, cubrir la demanda de productos, bienes o servicios tanto para sector público y privado.

- Se requiere que el sector de la EPS tenga estadísticas actualizadas y que hagan uso de herramientas tecnológicas o plataformas, para registrar el aporte al desarrollo local de un territorio en términos de empleo, aporte al PIB, requerimiento de mano de obra, acceso a factores productivos, información que servirá para medir el impacto e incidencia de la Economía Popular y Solidaria.

\section{Revisión bibliográfica}

Alburquerque, F. (2004). Desarrollo económico local y descentralización. CSIC, 162.

ASAMBLEA NACIONAL, E. (Nov. de 2011). Ley Organica de Economía Popular y Solidaria. LOEPS. Quito, Pichincha, Pichincha, Ecuador.

ASAMBLEA, N. D. (Noviembre de 2011). Constitución Política del Ecuador. Quito, Ecuador: Corporación de Estudio y Publicaciones.

Askunze, C. (2007). Economia Solidaria. Diccionario para el desarrollo, 107-113.

CEPAL, C. E. (2009). Informe Anual America Latina.

Coraggio, J. L. (2007). Es posible otra economía sin (otra) politica? Economía social, acción pública y política, 151-180.

Coraggio, J. L. (2010). La economia popular y solidaria en el Ecuador. Quito.

Coraggio, J. L. (2011). Economia Social y solidaria: el trabajo antes que el capital. Quito, Ecuador: ABYA-YALA.

Coraggio, J., Arancibia, M., \& Deux, M. (2010). Guia para mapeo y relevamiento de la economía popular solidaria en latinoamerica y caribe. Lima - Perú: CCFDTerre Solidaire y Ayuda en Accion.

Hernández, R., Fernández, C., \& Batista, P. (1991). MetodologIa de la investigación. McGraw-Hill.

Herrán, J. (2013). La economía popular y solidaria. Una práctica más que un concepto. RETOS.

IEPS. (2019). www.economiasolidaria.gob.ec. Obtenido de www.economiasolidaria.gob.ec.

Kline, R. B. (1998). Methodology in the social sciences.Principles and practice of structural equation modeling. New York: Kline LIbrary. 
Laville, G. y. (2009). Economia Solidaria. En g. L. Cattani, Diccionario Internacional de Outra Economia. Sao Paulo, Brasil: Almedina.

Lazarini, M. V. (2010). Hacia otra economía en América Latina: El papel de la economia social. Hacia otra economía en América Latina: El papel de la economia social, (págs. 29-34). Santiago de Compostela, España.

Lind, D., Marchal, W., \& Wathen, S. (2012). Estadística Aplicada a los negocios y a la economía. México: McGraw-Hill.

MIES, M. d. (2017). www.inclusión.gob.ec.

Muñoz, P. (2015). La Economía Popular y Solidaria, un eje clave del sistema económico. CHAKANA.

Oviedo, H., \& Campo-Arias, A. (2005). Aproximación al uso del coeficiente alfa de Cronbach. Revista Colombiana de Psiquiatria, 34 (4), 572-580.

Quijano, A. (2014). "Del polo marginal" a la "economia alternativa"? En Cuestiones y Horizontes: de la dependencia histórico-estructural a la colonia/descolonialidad del poder (págs. 215-229). Buenos Aires, Argentina: CLACSO.

Quijano, A. (2014). Colonialidad del poder, eurocentrismo y America latina. En A. Quijano, Cuestiones y Horizontes: de la dependencia histórico-estructural a la colonialidad/descolonialidad del poder (págs. 777-786). Buenos Aires, Argentina: CLACSO.

Razeto, L. (1984). Economía Solidaria y Mercado Democrático. Santiago de Chile, Chile: PET.

Razeto, L. (1993). Los caminos de la economia con solidaridad. Santiago de Chile, Chile: Ediciones Vivarium.

Razeto, L. (2007). La economia de solidaridad, concepto, realidad y proyecto. En J. L. Coraggio, La economía social desde la periferia. Contribuciones latinoamericanas (págs. 10-30). Buenos Aires, Argentina.

Saltos, J., \& Mayorga, M. (2004). La economía popular y solidaria: un estudio exploratorio del sistema en Ecuador con enfoque de control y fiscalización. Cofin Habana, 55 - 75.

SEPS. (2012). www.seps.gob.ec. Obtenido de www.seps.gob.ec.

SEPS. (2020). www.seps.gob.ec. Obtenido de www.seps.gob.ec

Singer, P. (2000). Economia Solidaria versus economía capitalista. Revista Semestral de Sociología, volumen XVI, No. 1-2, 19.

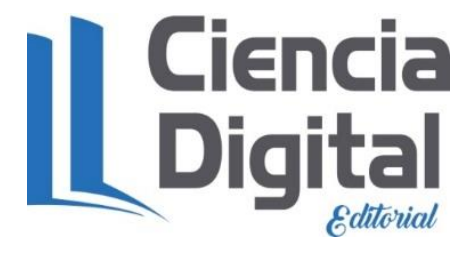




\section{PARA CITAR EL ARTÍCULO INDEXADO.}

Rosales Namicela, M. B., \& Carabajo Alvear, R. F. (2021). La economía popular y solidaria. una alternativa de desarrollo económico local en el caso del cantón cuenca. ConcienciaDigital, $\quad$ 4(1.2),

79-102. https://doi.org/10.33262/concienciadigital.v4i1.2.1581

\section{LCiencia}

El artículo que se publica es de exclusiva responsabilidad de los autores y no necesariamente reflejan el pensamiento de la Revista Conciencia Digital.

El artículo queda en propiedad de la revista y, por tanto, su publicación parcial y/o total en otro medio tiene que ser autorizado por el director de la Revista Conciencia Digital.

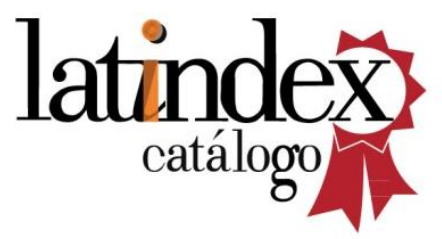

